\title{
DIVISION XII / COMMISSION 5 / WORKING GROUP DESIGNATIONS
}

\author{
CHAIR \\ MEMBERS
}

\author{
Marion Schmitz \\ Heinz J. Andernach, Suzanne Borde, \\ Kirk D. Borne, Anne P. Cowley, \\ Helene R. Dickel, Pascal Dubois, \\ John S. Gallagher, Françoise Genova, \\ Paul W. Hodge, Richard W. Hunstead, \\ Marie-Claire Lortet, Donald A. Lubowich, \\ Oleg Yu. Malkov, Tetsuya Nagata, \\ François Ochsenbein, Sean E. Urban, \\ Ethan T. Vishniac, Wayne H. Warren, \\ Norbert Zacharias
}

\section{PROCEEDINGS BUSINESS MEETING on 23 and 24 August 2006}

\section{Summary of three years activity}

At the 2003 Sydney IAU meeting, Marion Schmitz (Caltech, USA) took over the chair of the Commission 5 Working Group Designations, succeeding Helene Dickel. The Working Group Designations of IAU Commission 5 clarifies existing astronomical nomenclature and helps astronomers avoid potential problems when designating their sources. The most important function of WG Designations during the period 2003-2005 was overseeing the IAU REGISTRY FOR ACRONYMS (for newly discovered astronomical sources of radiation: see the website <http://cdsweb.u-strasbg.fr/cgi-bin/DicForm>) which is sponsored by the WG and operated by the Centre de Données de Strasbourg (CDS). The Clearing House, a subgroup of the WG, screens the submissions for accuracy and conformity to the IAU Recommendations for Nomenclature (<http://cdsweb.u-strasbg.fr/iau-spec.html>). From its beginning in 1997 through August 2006, there have been 132 submissions and 111 acceptances. Attempts to register asterisms, common star names, and suspected variable stars were rejected. The past three years saw 61 acronyms submitted with 50 of them being accepted. (GIRL - yes; WOMEN - no).

Assistance was provided for inquiries about nomenclature for planet-candidates discovered through microlensing events; a short history of naming stars (which has been posted on the Commission 5 web site <http://cdsweb.u-strasbg.fr/IAU/starnames.html>; and designations of Cosmic Microwave Background structures.

E-mail discussions included the changing of position-based names when new measurements or reprocessing indicates a different position. Jay Gallagher and Ethan Visniac were added to the e-mail distribution list for such discussions. Strong support from WG members was to retain the policy of NOT changing a designation once one had been established.

E-mail discussions concerning follow-up observations of an area of sky by different telescopes. Suggestions from the WG were split between a format of: "Area of Sky" "Telescope" designation and "Telescope" "Area of Sky". Examples include: COSMOSVLA, HDFSST, GOODS-MUSIC, vs. CXOHDF, CXOM31.

Lanie Dickel and Ray Norris submitted several short newspaper articles for the IAU newspaper Nuncio Sidereo III. Thank you, both, for helping spread our important messages to the IAU community. 